\title{
Are level of IL-13 and IL-4 predictive for formation of chronic inflammation in children with asthma?
}

\begin{abstract}
Introduction: Asthma diagnosis in young children may represent a clinical challenge. There are no standard prognostic and diagnostic methods. The aim of the study was to evaluate the clinical and prognostic assessment of IL-4 and IL-13 concentrations in children with recurrent wheezing.

Material and methods: The study included 96 children with recurrent wheezing. 81 patients were diagnosed as transient wheezing, 15 patients with asthma, and 25 healthy children were selected as controls. The concentrations of IL-4 and IL-13 were analyzed in exhaled breath condensate (EBC) using an enzyme-linked immunosorbent assay (ELISA). Data analysis was performed using Statsoft Statistica Version 8 (Tulsa, OK) and the statistical program MedCalc version 17.2.

Results: Both IL-4 and IL-13 concentrations were significantly higher in DDA $(21.13 \mathrm{pg} / \mathrm{mL}, 26.13 \mathrm{pg} / \mathrm{mL}$, respectively) and TW $(13.86 \mathrm{pg} / \mathrm{mL}, 18.3 \mathrm{pg} / \mathrm{mL}$, respectively) groups as compared to healthy controls $(3.37 \mathrm{pg} / \mathrm{mL}, 16.35 \mathrm{pg} / \mathrm{mL}$, respectively; $p<0.001$ ), and the highest rates were observed in children with diagnosed asthma ( $p<0.001$, DDW vs TW, respectively). IL-4 concentration higher than $18.45 \mathrm{pg} / \mathrm{mL}$ (with sensitivity $86.7 \%$ and specificity $80 \%$ ) and IL-13 concentration higher than $20.17 \mathrm{pg} /$ $/ \mathrm{mL}$ (with sensitivity $100 \%$ and specificity $76.7 \%$ ) in EBC in children with wheezing recurrence can be considered as a possible predictor of asthma development.

Conclusions: The concentration of the anti-inflammatory cytokines IL-4 and IL-13 were significantly increased in children with recurrent wheezing and the highest rates were found in asthma developing children. The concentrations of IL-4 and IL-13 in children with wheezing can be considered as a possible predictor of asthma development.
\end{abstract}

Key words: asthma, inflammation, IL-4, IL-13, children

Adv Respir Med. 2020; 88: 320-326

\section{Introduction}

Asthma is one of the most common chronic diseases in children and young adults [1-5]. The number of severe and uncontrolled forms of asthma has increased, leading to greater disability and mortality among patients [1-2]. The debut of the disease takes place in childhood [3-5]. Accurate diagnosis of asthma in this age cohort may represent a significant clinical challenge $[1,3,6]$. Firstly, the clinical signs of wheezing in children can be caused by many diseases, among which, besides asthma, there are hereditary and birth defects with metabolic processes, pathology of the gastroesophageal zone, congenital and acquired defects of the heart and main vessels and many others that should be included in the differential diagnostics [3-5, 7]. Secondly, in children under 6 years of age, there are limitations in the study of lung function. Therefore, in this cohort, diagnosis is based mainly on clinical and anamnestic data [6-8]. So, the global medical problem is to expand the diagnostic possibilities of early detection of asthma in children.

The pathogenesis of the disease, namely chronic inflammation, is a systemic process, but it damages mainly the respiratory tract $[8,9]$. At the beginning of the formation of inflammation, the mechanisms do not depend on the type of the damage (allergic, infectious, etc.) and have general signs of action of cytokine complexes [10-13]. Several proinflammatory (IL1, IL-6, IL-8, TNF- $\alpha$,

Address for correspondence: Valeriia Malakhova, Kharkiv National Medical University, Kharkiv, Ukraine; e-mail: malakhovavaleriia@gmail.com

DOI: 10.5603/ARM.a2020.0108

Received: 17.02.2020

Copyright (C) 2020 PTChP

ISSN 2451-4934 
GM-CSF) and anti-inflammatory cytokines (IL-4, IL-10, IL-13, TGF-b) are synthesized [11-13]. It is known that IL-4 function is to induce increased concentration of IgE, and IL-13 is a unique, Th2-linked, cytokine that interacts with B cells and thus regulates inflammatory and immune responses [14-16]. Recent studies have found an increase in serum concentration of these anti-inflammatory cytokines. They are not only able to induce the inflammatory process, but also support it, leading to a chronic activity. These interleukins that characterize chronic inflammation lead to airway remodeling and hyperplasia of the muscular system of the bronchopulmonary system [17]. Thus, it is advisable to study the concentration of these indicators directly in the inflammation locus.

The study of mediators and markers of inflammation released by damage of the respiratory system is possible due to the procedure of bronchoalveolar lavage or induced sputum [18]. However, because of the fact that these studies are related to invasive intervention, they cannot be repeated in a short period of time, especially in pediatric patients. Considering non-invasive methods that can be used in children, and to be able to reflect the state of local inflammation of the respiratory system, the exhaled breath condensate (EBC) seems to be optimal option [18]. It is a promising source of biomarkers of lung disease. EBC represents a matrix in which biomarkers can be identified [18, 19]. Till date interleukins' levels in EBC, especially in cohorts of young children have been rarely evaluated. In this study, we conducted clinical and prognostic evaluation of interleukin 4 and interleukin 13 concentration in EBC children with recurrent wheezing episodes.

\section{Material and methods}

\section{Study design}

This was a longitudinal cohort study of children with recurrent wheezing from February 2015 to November 2019 who were treated at a children's hospital. The study consisted of two stages. In the first phase (recruitment, February 2015 - May 2015), among the 305 children who were admitted to the hospital with wheezing, 126 children between the ages of 1.5 and 6 , who met the inclusion criteria and did not fulfill exclusion criteria were included to the study. Inclusion criteria: signing informed consent by the patient's parents; patient's age from 1.5 to 6 years; current wheezing episode at recruitment is third and more. Exclusion criteria: congenital and chronic cardiopulmonary or neurological disease; hereditary diseases that lead to changes in the functioning of the respiratory tract, including cystic fibrosis; proven immune deficiency; proven or suspected acute or chronic bacterial infection, including infection of the oral cavity and respiratory tract; suspected or confirmed gastroesophageal diseases; previous treatment with antileukotriene drugs or systemic corticosteroids. The patients underwent specific treatment according to accepted recommendations [20]. All study subjects underwent clinical medical history review, physical examination and laboratory assessment. Children were also examined for IL-4 and IL-13 concentration in the exhaled breath condensate. The study of anti-inflammatory cytokines was conducted on the first day of the disease in the presence of clinical manifestations of wheezing.

The control group comprised 25 healthy children (of similar age/gender) without any signs of chronic or acute diseases for the previous three months, presented to Hospital for routine health control or vaccination. The parents of all patients and controls were informed about the study objectives and written informed consent was obtained before inclusion in the study.

Second stage (follow-up, May 2015-September 2019). The patients were called for re-examination at the age of 5-6 years. The inclusion and exclusion criteria in the study were re-evaluated. In the second stage, 96 children met the inclusion criteria and did not satisfy exclusion criteria. Anamnestic data for this period were studied. An examination was carried out to verify the diagnosis of asthma at the time of re-examination. The diagnosis was based on the GINA 2019 criteria and included symptoms (cough and wheezing) for more than 10 days during the upper respiratory tract infection, more than 3 episodes of wheezing per year, the child coughed between episodes, and there were present atopic dermatitis or food allergy history or family history of asthma [20]. An objective assessment of the severity of respiratory failure and differential diagnosis of obstructive and possible restrictive respiratory disorders were performed. The patients were divided into groups depending on the asthma diagnosed during this period or diagnosed during the visit. The first group included 81 transient wheezing (TW) children who stopped wheezing symptom at follow-up. The second group included 15 patients with doctor-diagnosed asthma (DDA). 


\section{Exhaled breath condensate: technical and diagnostic aspects}

The device for EBC receiving was designed using a portable glass tube in accordance with material collection standards. The glass tube was cooled, surrounded by a mixture of refrigerants to obtain a temperature that reached from $-5^{\circ} \mathrm{C}$ to $-10^{\circ} \mathrm{C}$. There, exhaled air in the form of droplets was converted to EBC [18, 21]. After collection, the glass tube was detached, and the sample was stored immediately at $-70^{\circ} \mathrm{C}$. The device has a manual control of condensing temperature and cleaning requirements of the tool between consequent trials. All collections were done between 8:30 and 09:30 a.m. The subjects wore a nose clip, which prevented contamination of the material with excretions from the nasal mucosa, and prevented the possibility of inhaling or exhaling through the nasal cavity. For children over three years of age, condensate was collected through systems with a single-use mouthpiece that was connected to a one-way exhalation valve to prevent the effects of inhaling the condensate. For subjects younger than two years, an exhalation valve with an inhalation mask was used.

\section{Measurement of IL-4 and IL-13 in exhaled breath condensate}

IL-4 in EBC were analyzed by the ELISA technique using commercial kits (Human IL-4, "Vector Best Ukraine", catalog number: A-8754), and IL-13 concentration were analyzed by the ELISA technique using commercial kits (Human IL-13, eBioscience (Bender MedSystems), catalog number: BMS231-3, USA) according to the manufacturer's instructions.

\section{Statistical analysis}

All statistical analyses were performed using StatSoft STATISTICA Version 8 package program (Tulsa, OK) and MedCalc statistical Software Version 17.2. Shapiro-Wilk's test was used, and histogram and q-q plots were examined to assess the normality. As the sample distribution was different from the normal, the median (Me) and the interquartile range were determined (Lq - lower quartile; Uq - upper quartile). Fisher's exact test, $\chi 2$ was used to calculate two relative indicators. Nonparametric Mann-Whitney test (MW) was applied to compare the two samples and Wilcoxon (T) nonparametric test was used to compare the two dependent samples. The difference between the two param- eters was considered statistically significant at $p<0.05$. The correlation between parameters was determined using the Spearman rank correlation analysis (r); $\mathrm{p}<0.05$ was considered to indicate a statistically significant difference. Receiver operating characteristic (ROC) curves were drawn for variables to determine the optimal «cut-off» values to predict an endpoint. The endpoint of this study is the formation of chronic inflammation in children with recurrent wheezing. The cut-off point of each variable and sensitivity, specificity, positive likelihood ratio (+LR), negative likelihood ratio (LR) of this "cut-off" point was obtained using the Youden index. To determine the most reliable screening tool among these four variables, pairwise comparison of these variables was performed by determining the differences between area under the curve using the Hanley and McNeil method.

\section{Ethics approval and consent to participation}

The planned clinical studies were carried out after receiving approval by the local ethics committee (date: February 1, 2015; number: 2015/01) and were conducted in accordance with the principles of the Helsinki Declaration, amended in October 2013.

\section{Results}

\section{General information}

Of the 96 children with recurrent wheezing enrolled to the study, the majority were diagnosed with transient wheezing (TW) (as they stopped wheezing at follow-up), and a minority developed asthma (DDA). These groups accounted for $84.38 \%$ and $15.62 \%$, respectively. No significant difference was found when comparing age and gender groups. Anamnestic factors such as pregnancy, cesarean delivery, and frequent manifestations of viral infections of the upper respiratory tract, presence of pets, passive smoking were not statistically different between those groups. In regard of allergic history, atopic dermatitis, food allergy, allergic rhinitis, the presence of allergic diseases and asthma in relatives, wheezing in the first year of life were likely to be more frequent in patients with DDA. Elevated eosinophil level and high IgE level were also significantly increased in patients with DDA (Table 1).

\section{IL-4 and IL-13 concentration}

IL-4 concentrations were significantly higher in DDA (21.13 pg/mL) and TW (13.86 pg/mL) groups 
Table 1. General information of anamnesis data [\%(n)]

\begin{tabular}{|c|c|c|c|}
\hline Sign & $\begin{array}{c}\text { TW } \\
(n=81)\end{array}$ & $\begin{array}{c}\text { DDA } \\
(n=15)\end{array}$ & $\mathbf{P}$ \\
\hline Gender, $\mathrm{M} / \mathrm{F}$ & $48 / 33$ & $10 / 5$ & $p>0.05$ \\
\hline Age, years at recruitment $\mathrm{Me}(\mathrm{Lq} ; \mathrm{Uq})$ & $2.58(1.55 ; 4.10)$ & $2.00(1.50 ; 3.11)$ & $p>0.05$ \\
\hline Age, years at follow-up Me $(\mathrm{Lq} ; \mathrm{Uq})$ & $7.09(6.00 ; 9.90)$ & $6.50(6.00 ; 7.80)$ & $p>0.05$ \\
\hline Caesarean section & $42.2 \%(35 / 81)$ & $33.3 \%(5 / 15)$ & $p>0.05$ \\
\hline Gestation period & $39(35 ; 41)$ & $38(35 ; 40)$ & $p>0.05$ \\
\hline Presence of atopic dermatitis in children & $39.5 \%(32 / 81)$ & $73.3 \%(11 / 15)$ & $p=0.0227$ \\
\hline Presence of food allergy in children & $35.8 \%(29 / 81)$ & $80.0 \%(12 / 15)$ & $p=0.0033$ \\
\hline Present of allergic rhinitis in children & $2.5 \%(2 / 81)$ & $46.7 \%(7 / 15)$ & $\mathrm{p}<0.001$ \\
\hline Present of allergic disease in relatives & $3.7 \%(3 / 81)$ & $60.0 \%(9 / 15)$ & $P<0.001$ \\
\hline Presence of asthma in relatives & $2.5 \%(2 / 81)$ & $53.3 \%(8 / 15)$ & $P<0.001$ \\
\hline Onset of wheezing of the first year of life & $48.1 \%(39 / 81)$ & $80.0 \%(12 / 15)$ & $p=0.0267$ \\
\hline Current wheezing episode at recruitment & $4(3 ; 4)$ & $5(3 ; 5)$ & $p>0.05$ \\
\hline Current pet ownership at recruitment & $27.2 \%(22 / 81)$ & $26.7 \%(4 / 15)$ & $p>0.05$ \\
\hline Repeated upper respiratory tract infection & $85.2 \%(69 / 81)$ & $80.0 \%(12 / 15)$ & $p>0.05$ \\
\hline Passive smoking & $27.2 \%(22 / 81)$ & $26.7 \%(4 / 15)$ & $p>0.05$ \\
\hline High eosinophil blood & $8.7 \%(7 / 81)$ & $60.0 \%(9 / 15)$ & $\mathrm{p}<0.001$ \\
\hline IgE increase, IU/ml & $25.9 \%(21 / 81)$ & $86.7 \%(13 / 15)$ & $\mathrm{p}<0.001$ \\
\hline
\end{tabular}

DDA — doctor diagnosed asthma; TW — transient wheeze

as compared to healthy controls $(3.37 \mathrm{pg} / \mathrm{mL}$; $\mathrm{p}<0.001$ ), and the highest rates were observed in children with diagnosed asthma $(\mathrm{p}<0.001$, DDW vs TW, respectively) (Table 2). Similarly, IL-13 concentrations were significantly higher in DDA $(26.13 \mathrm{pg} / \mathrm{mL})$ and TW $(18.3 \mathrm{pg} / \mathrm{mL})$ groups as compared to healthy controls $(16.35 \mathrm{pg} / \mathrm{mL}$; $\mathrm{p}<0.001$ ), and the highest rates were observed in children with diagnosed asthma $(\mathrm{p}<0.001$, DDW vs TW, respectively).

\section{Correlation between IL-4 and IL-13 parameters}

The correlation between IL-4 and IL-13 concentration of all children with recurrent wheezing was found ( $\mathrm{r}=+0.37, \mathrm{p}<0.001)$. The relationship between those biomarkers in children with DDA was $\mathrm{r}=+0.74, \mathrm{p}<0.001$, and in TW cohort $\mathrm{r}=+0.17, \mathrm{p}<0.001$.

\section{Prognostic criteria for IL-4 and IL-13}

To determine the prognostic significance of the concentration of IL-4 and IL-13, ROC curve analysis was performed. The relationship between cytokine level during the first episodes of wheezing and the development of asthma was determined. The cut-off value for the concentra- tion of IL-4 higher than $18.45 \mathrm{pg} / \mathrm{mL}$ resulted in specificity of $80 \%$ and sensitivity of $86.7 \%$, and for IL-13, concentration higher than $20.17 \mathrm{pg} / \mathrm{mL}$ resulted in specificity of $76.7 \%$ and sensitivity of $100 \%$ for the predefined endpoint, namely the formation of chronic asthma (Figure 1).

\section{Discussion}

This study found that among children with recurrent wheezing, a greater proportion of patients had transient wheezing and only $15.62 \%$ developed asthma during follow-up. There are many studies that describe the possible contributing factors in children with recurrent wheezing for formation of asthma. Several phenotypes are considered as the debut of the disease. The proportion of such children ranges from $13 \%$ to $16 \%$ according to various sources [6, 22-24]. This fact has been confirmed in our study.

In our study, an increased concentration of IL-4 in the exhaled breath condensate of children with recurrent wheezing in the onset of clinical manifestations was determined. The highest rates were found in children who developed asthma during the follow-up. IL-4 is known to stimulate IgE production, promote eosinophil accumulation in peripheral blood and tissues [14-17, 25-26]. 
Table 2. IL-4 and IL-13 levels in children with recurrent wheezing during the onset of clinical manifestations (Me [Lq; Uq])

\begin{tabular}{|c|c|c|c|}
\hline & TW $(n=81)$ & $\begin{array}{c}\text { DDA } \\
(n=15)\end{array}$ & Control \\
\hline \multirow[t]{2}{*}{$\begin{array}{c}\text { IL-4, } \\
\text { Me (Lq; Uq) }\end{array}$} & $\begin{array}{c}13.86 \mathrm{pg} / \mathrm{mL} \\
(10.16 ; 16.48)\end{array}$ & $\begin{array}{l}21.13 \mathrm{pg} / \mathrm{mL} \\
(19.28 ; 1.54)\end{array}$ & $\begin{array}{l}3.37 \mathrm{pg} / \mathrm{mL} \\
(2.95 ; 3.62)\end{array}$ \\
\hline & $\begin{array}{c}\mathrm{KW} \text { by } \mathrm{R} \\
\mathrm{MW}: \mathrm{p} 1-2<0.001\end{array}$ & $\begin{array}{l}<0.001 \\
\quad \text { p2- contr }\end{array}$ & \\
\hline $\begin{array}{c}\text { IL-13, } \\
\text { Me (Lq; Uq) }\end{array}$ & $\begin{array}{c}18.30 \mathrm{pg} / \mathrm{mL} \\
(16.11 ; 20.17)\end{array}$ & $\begin{array}{c}26.13 \mathrm{pg} / \mathrm{mL} \\
(24.12 ; 31.05)\end{array}$ & $\begin{array}{c}16.35 \mathrm{pg} / \mathrm{mL} \\
(14.86 ; 17.45)\end{array}$ \\
\hline
\end{tabular}

DDA — doctor diagnosed asthma; KW — Kruskal-Wallis test; Me (Lq; Uq) — median (lower quartile; upper quartile); MW— Mann-Whitney test; $\mathrm{p}$ significant with the Bonferroni correction; TW - transient wheeze

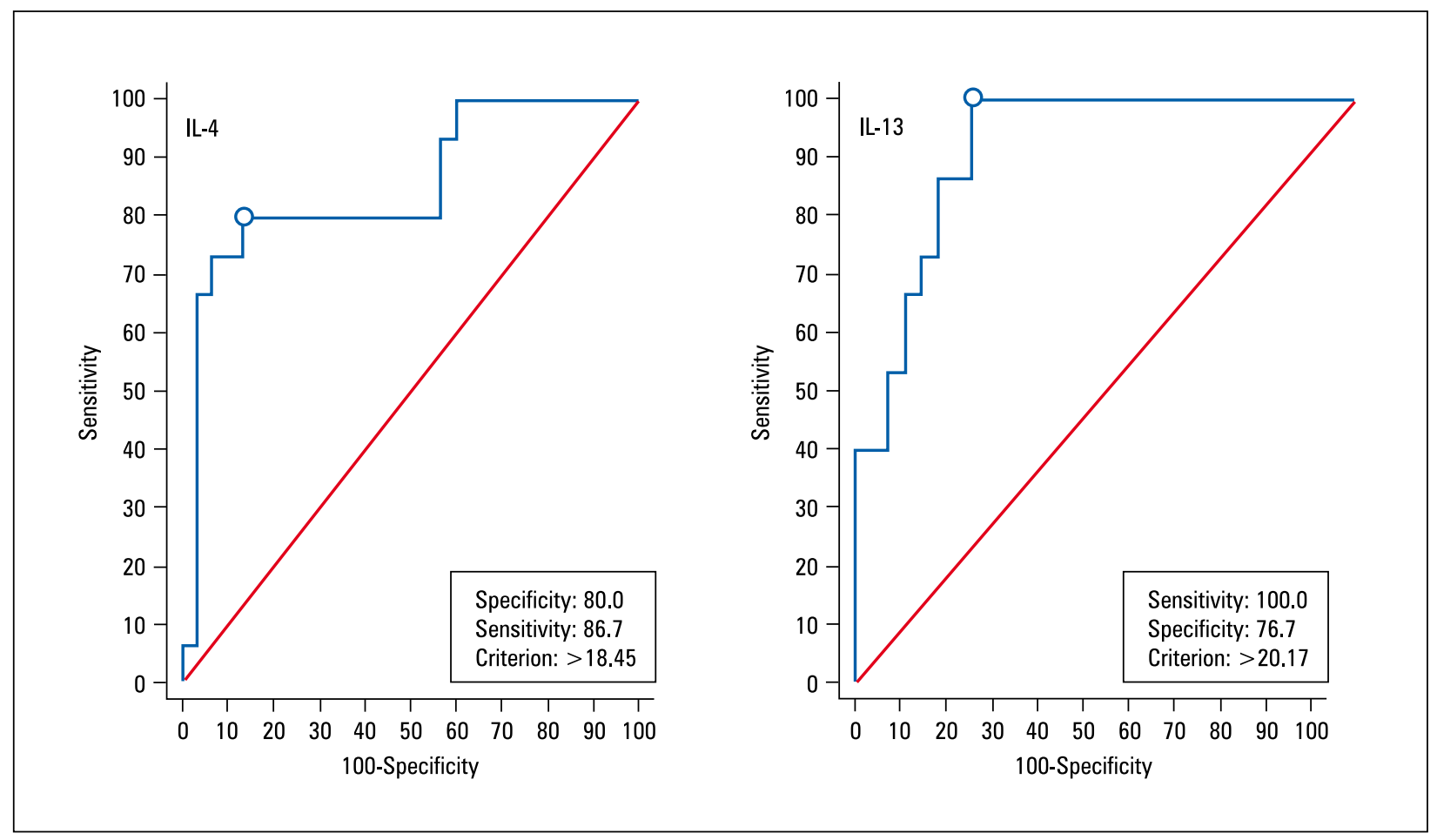

Figure 1. ROC curves for IL-4 and IL-13 as a biomarker of asthma development in children with wheezing episode

As a result, IL-4 is regarded as a cytokine that has a direct effect on the development of allergic inflammation [14-17]. An increased concentration of this cytokine has been shown to indicate the presence of allergic inflammation in patients. There are studies demonstrating changes in serum IL-4 concentration in atopic dermatitis in children [25], adults, and adolescents with asthma [14, 17]. Recent researches have proved that IL-4 concentration increase in the EBC of adult asthma patients, and IL-4 is considered as a marker of chronic allergic inflammation [26]. In our study, children up to 6 years of age were examined, but IL-4 was also elevated, which was likely to indicate an allergic nature of inflammation of the bronchopulmonary system. An increased IL-4 concentration in the exhaled breath condensate can be considered as a manifestation of an active inflammatory process, directly in the inflammation locus.

We found that in children with recurrent wheezing, in the onset of clinical manifestations, IL-13 concentration in exhaled breath condensate was also increased as compared to healthy controls. The highest levels were found in children who developed asthma. IL-13 is a Th2-linked cytokine and regulates the body's inflammatory and immune responses. It stimulates the production 
of B cells and IgE, and inhibits the formation of inflammatory cytokines [14-17]. Recent studies have reported an increased IL-13 concentration in serum in atopic diseases in children [17, 25]. There are data that confirmed an elevated IL13 concentration in the serum in adolescents and adults asthma $[14,17]$. These studies suggest that changes in the concentration of this cytokine indicate the presence of allergic inflammation. We have also regarded it as a manifestation of allergic inflammation, as well as activity of the process in the respiratory system, taking into account the detection of indicators in the locus of damage.

Our study evaluated the relationship between IL-4 and IL-13 in EBC children less than 6 years of age and established direct correlation between indicators. Similarly, significant correlation was found in children who developed asthma. The correlations of IL-13 and IL-4 were made taking into account the presence of similar pathways that are involved in the synthesis of IgE, activation of eosinophils, mucus secretion [14-16]. Recent studies have demonstrated these relationships in adult patients with allergic airway disease $[17,25]$, where IL-4 has been identified as the first cytokine to be produced by mast cells and responsible for promoting the production of IL13 of mast cells [15]. Our study confirms the relationship between these cytokines in the presence of allergic inflammation in children.

The ROC analysis determined the predictive concentrations of IL-4 and IL-13 for development of asthma in children with wheezes. The criterion of IL-4 > $18.45 \mathrm{pg} / \mathrm{mL}$ and IL-13 $>20.17 \mathrm{pg} / \mathrm{mL}$ were found to have prognostic significance, with high specificity and sensitivity rates in asthma formation in children with recurrent wheezing.

Non-invasive techniques are used to investigate the health status of adults and children. There are several non-invasive techniques for the determination of respiratory gases in exhaled air and compounds dissolved in exhaled lung secretion. There are studies using Electronic Noses (E-noses) that can play a potential role in the screening and analysis of various respiratory and systemic diseases [27-29]. The EBC was chosen for our study.

This study has several limitations. Firstly, about $45 \%$ of children were with comorbid diseases, such as atopic dermatitis or food allergy, which may result in higher concentration of IL-4 and IL-13 both in serum and EBC. Thus, the concentration of these cytokines obtained in children with asthma could have higher rates due to concomitant allergic diseases [14, 25]. However, there is no evidence-based data that concentration of
IL-4 or IL-13 can increase in EBC in children with comorbid allergic diseases. Substances in the EBC are in large dilution, as the excretory fluid covers both the alveolar epithelial layer and the mucous layer of the respiratory tract. There is no existing model that allows collecting all the exhalation, since the loss of moisture depends on the humidity and temperature of the environment. These factors are likely to have an influence on the obtained results [18-19, 21, 26]. Another limitation of the study is that asthma is a heterogeneous disease and has many phenotypes [9, 22, 30-31]. There are types that have neutrophil and paucigranulocytic phenotype in their mechanism of development. The most common is the eosinophilic type of inflammation, which is associated with eosinophilic cellular infiltration and thickening of the basement membrane area. The performed studies and the selected cytokines reflect just this type of inflammation. This study was conducted taking into account only the eosinophilic phenotype, which is the most frequent in children.

In conclusion, among patients presenting with recurrent wheezing, approximately in every sixth child, the disease has been transformed into asthma. The concentrations of the IL-4 and IL-13 in exhaled breath condensate were significantly increased in children with manifestation of wheezing, and the highest rates were found in asthma developing children. Both cytokines are involved in the regulation of allergic inflammatory processes in the body, and have an influence on the respiratory tract, as it was detected basing on IL-4 and IL-13 concentration in the exhaled breath condensate. IL-4 concentration higher than $18.45 \mathrm{pg} / \mathrm{mL}$ and IL-13 concentration higher than $20.17 \mathrm{pg} / \mathrm{mL}$ in exhaled breath condensate in children with wheezing recurrence can be considered as a possible predictor of asthma development.

\section{Conflict of interest}

None declared.

\section{References:}

1. Guo J, Zhu W, Wang H, et al. Risk factors and prognosis of recurrent wheezing in Chinese young children: a prospective cohort study. Allergy, Asthma \& Clinical Immunology. 2019; 15(1), doi: 10.1186/s13223-019-0351-4.

2. Butov D, Makieieva N, Vasylchenko Y, et al. Interrelationship of endothelial function parameters in children with bronchial asthma in exacerbation and remission. Advances in Respiratory Medicine. 2015, doi: 10.5603/arm.a2019.0002.

3. Soh JiE, Kim KM, Kwon JW, et al. Recurrent wheeze and its relationship with lung function and airway inflammation in preschool children: a cross-sectional study in South Korea. BMJ Open. 2017; 7(10): e018010, doi: 10.1136/ bmjopen-2017-018010, indexed in Pubmed: 28993393. 
4. Hendaus MA, Jomha FA, Ehlayel M. Allergic diseases among children: nutritional prevention and intervention. Ther Clin Risk Manag. 2016; 12: 361-372, doi: 10.2147/TCRM.S98100, indexed in Pubmed: 27022267.

5. Castro-Rodriguez JA, Forno E, Rodriguez-Martinez CE, et al. Risk and protective factors for childhood asthma: what is the evidence? J Allergy Clin Immunol Pract. 2016; 4(6): 1111-1122, doi: 10.1016/j.jaip.2016.05.003, indexed in Pubmed: 27286779.

6. Al-Shamrani A, Bagais $\mathrm{K}$, Alenazi A, et al. Wheezing in children: Approaches to diagnosis and management. Int J Pediatr Adolesc Med. 2019; 6(2): 68-73, doi: 10.1016/j.ijpam.2019.02.003, indexed in Pubmed: 31388550.

7. Liu Lu, Pan Y, Zhu Y, et al. Association between rhinovirus wheezing illness and the development of childhood asthma: a meta-analysis. BMJ Open. 2017; 7(4): e013034, doi: 10.1136/ bmjopen-2016-013034.

8. Kalliola S, Malmberg LP, Malmström K, et al. Airway hyperresponsiveness in young children with respiratory symptoms: A five-year follow-up. Ann Allergy Asthma Immunol. 2019; 122(5): 492-497, doi: 10.1016/i.anai.2019.02.025, indexed in Pubmed: 30831260.

9. Agache IO. From phenotypes to endotypes to asthma treatment. Curr Opin Allergy Clin Immunol. 2013; 13(3): 249-256, doi: 10.1097/ACI.0b013e32836093dd, indexed in Pubmed: 23587683.

10. Kawayama T, Kinoshita T, Matsunaga K, et al. Role of regulatory $\mathrm{T}$ cells in airway inflammation in asthma. Kurume Med J. 2018; 64(3): 45-55, doi: 10.2739/kurumemedj.MS6430001, indexed in Pubmed: 29553094.

11. Kubo M. Innate and adaptive type 2 immunity in lung allergic inflammation. Immunol Rev. 2017; 278(1): 162-172, doi: 10.1111/imr.12557, indexed in Pubmed: 28658559.

12. Gao H, Ying S, Dai Y. Pathological Roles of Neutrophil-Mediated Inflammation in Asthma and Its Potential for Therapy as a Target. J Immunol Res. 2017; 2017: 3743048, doi: 10.1155/2017/3743048, indexed in Pubmed: 29359169.

13. Ray A, Kolls JK. Neutrophilic inflammation in asthma and association with disease severity. Trends Immunol. 2017; 38(12): 942-954, doi: 10.1016/j.it.2017.07.003, indexed in Pubmed: 28784414 .

14. Bagnasco D, Ferrando M, Varricchi G, et al. A critical evaluation of anti-il-13 and anti-il-4 strategies in severe asthma. Int Arch Allergy Immunol. 2016; 170(2): 122-131, doi 10.1159/000447692, indexed in Pubmed: 27637004.

15. McLeod JJA, Baker B, Ryan JJ. Mast cell production and response to IL-4 and IL-13. Cytokine. 2015; 75(1): 57-61, doi: 10.1016/i.cyto.2015.05.019, indexed in Pubmed: 26088754.

16. Zhu J. T helper 2 (Th2) cell differentiation, type 2 innate lymphoid cell (ILC2) development and regulation of interleukin-4 (IL-4) and IL-13 production. Cytokine. 2015; 75(1): 14-24, doi: 10.1016/j.cyto.2015.05.010, indexed in Pubmed: 26044597.

17. Gour N, Wills-Karp M. IL-4 and IL-13 signaling in allergic airway disease. Cytokine. 2015; 75(1): 68-78, doi: 10.1016/j. cyto.2015.05.014, indexed in Pubmed: 26070934.

18. Davis MD, Montpetit AJ. Exhaled Breath Condensate: An Update. Immunol Allergy Clin North Am. 2018; 38(4): 667-678, doi: 10.1016/i.iac.2018.06.002, indexed in Pubmed: 30342587.
19. Ghio AJ, Soukup JM, McGee J, et al. Iron concentration in exhaled breath condensate decreases in ever-smokers and COPD patients. J Breath Res. 2018; 12(4): 046009, doi: 10.1088/17527163/aad825, indexed in Pubmed: 30079894.

20. The Global Strategy for Asthma Management and Prevention, Global Initiative for Asthma (GINA) 2019. Available at: www ginasthma org. [Last accessed at: 01.06.2020].

21. Winters BR, Pleil JD, Angrish MM, et al. Standardization of the collection of exhaled breath condensate and exhaled breath aerosol using a feedback regulated sampling device. J Breath Res. 2017; 11(4): 047107, doi: 10.1088/1752-7163/aa8bbc, indexed in Pubmed: 28894051.

22. Depner M, Fuchs O, Genuneit J, et al. PASTURE Study Group. Clinical and epidemiologic phenotypes of childhood asthma. Am J Respir Crit Care Med. 2014; 189(2): 129-138, doi: 10.1164/rccm.201307-1198OC, indexed in Pubmed: 24283801.

23. Granell R, Henderson AJ, Sterne JA. Associations of wheezing phenotypes with late asthma outcomes in the Avon Longitudinal Study of Parents and Children: A population-based birth cohort. J Allergy Clin Immunol. 2016; 138(4): 1060-1070. e11, doi: 10.1016/j.jaci.2016.01.046, indexed in Pubmed: 27106203.

24. Ren CL, Esther CR, Debley JS, et al. ATS Ad Hoc Committee on Infants with Recurrent or Persistent Wheezing. Official American Thoracic Society Clinical Practice Guidelines: diagnostic evaluation of infants with recurrent or persistent wheezing. Am J Respir Crit Care Med. 2016; 194(3): 356-373, doi: 10.1164/rccm.201604-0694ST, indexed in Pubmed: 27479061.

25. Matsunaga MC, Yamauchi PS. IL-4 and IL-13 inhibition in atopic dermatitis. J Drugs Dermatol. 2016; 15(8): 925-929, indexed in Pubmed: 27537991.

26. Chi CH, Liao JP, Zhao YN, et al. Effect of inhaled budesonide on interleukin-4 and interleukin-6 in exhaled breath condensate of asthmatic patients. Chinese Medical Journal. 2016; 129(7): 819-823, doi: 10.4103/0366-6999.178962.

27. Behera B, Joshi R, Anil Vishnu GK, et al. Electronic nose: a non-invasive technology for breath analysis of diabetes and lung cancer patients. J Breath Res. 2019; 13(2): 024001, doi: 10.1088/1752-7163/aafc77, indexed in Pubmed: 30620934.

28. Wilson AD. Application of electronic-nose technologies and voc-biomarkers for the noninvasive early diagnosis of gastrointestinal diseases . Sensors (Basel). 2018; 18(8), doi: 10.3390/ s18082613, indexed in Pubmed: 30096939.

29. Behera B, Joshi R, Anil Vishnu GK, et al. Electronic nose: a non-invasive technology for breath analysis of diabetes and lung cancer patients. J Breath Res. 2019; 13(2): 024001, doi: 10.1088/1752-7163/aafc77, indexed in Pubmed: 30620934.

30. Ozdemir C, Kucuksezer UC, Akdis M, et al. The concepts of asthma endotypes and phenotypes to guide current and novel treatment strategies. Expert Rev Respir Med. 2018; 12(9): 733-743, doi: 10.1080/17476348.2018.1505507, indexed in Pubmed: 30084271

31. Braido F, Tiotiu A, Kowal K, et al. Phenotypes/endotypes-driven treatment in asthma. Curr Opin Allergy Clin Immunol. 2018; 18(3): 184-189, doi: 10.1097/ACI.0000000000000440, indexed in Pubmed: 29601354. 